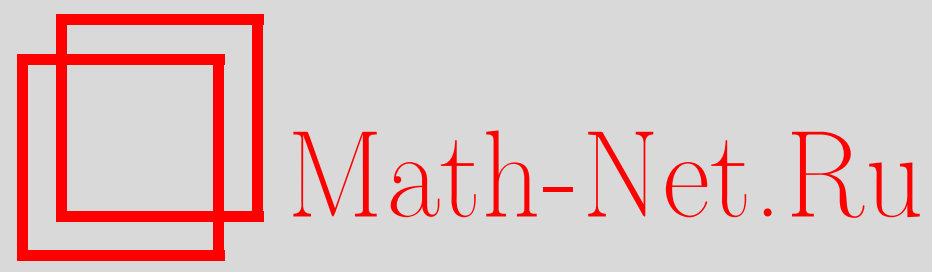

А. Г. Нурмиев, Д. И. Артамкин, Орбиты и инварианты кубических матриц третьего порядка с симметричными слоями, Матем. заметки, 2002, том 72, выпуск 4, 483-489

DOI: https://doi.org/10.4213/mzm437

Использование Общероссийского математического портала Math-Net.Ru подразумевает, что вы прочитали и согласны с пользовательским соглашением http://www.mathnet.ru/rus/agreement

Параметры загрузки:

IP : 54.157 .27 .8

26 апреля 2023 г., 13:29:38

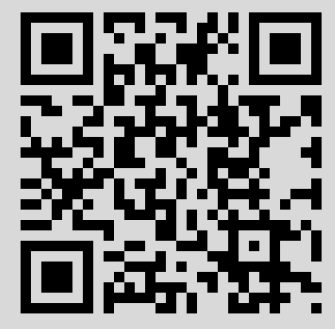




\title{
ОРБИТЫ И ИНВАРИАНТЫ КУБИЧЕСКИХ МАТРИЦ ТРЕТЬЕГО ПОРЯДКА С СИММЕТРИЧНЫМИ СЛОЯМИ
}

\author{
Д. И. Артамкин, А.Г. Нурмиев
}

\begin{abstract}
В заметке классифицированы орбиты и описаны образующие алгебры инвариантов действия, являющегося тензорньм произведением тавтологического действия унимодулярной группы трехмерного пространства и второй симметрической степени тавтологического действия унимодулярной группы трехмерного пространства.

Библиография: 5 названий.
\end{abstract}

1. В данной заметке классифицированы орбиты и описаны образуюшие алгебры инвариантов естественного представления групшы $H=\mathrm{SL}\left(V_{1}\right) \times \mathrm{SL}\left(V_{2}\right)$ в пространстве $U=S^{2} V_{1} \otimes V_{2}$, где $V_{i}, i=1,2$, - трехмерное комплексное векторное пространство. Отметим, что алгебра инвариантов данного представления свободна и степени образуюших суть 6, 12 (см. [1]). В работе [1] указана также группа Вейля для данного представления в смысле теории $\theta$-групп.

2. Введем основные обозначения и соглашения. Если $V$ - векторное пространство, то $V^{*}$ - двойственное векторное пространство. Для произвольной групшы Ли $L$ через $\operatorname{Lie}(L)$ обозначается ее алгебра Ли. Положим $V=V_{1} \oplus V_{1} \oplus V_{2}$, где $V_{1}, V_{2}$ те же, что и в п. 1. В дальнейшем будем отождествлять $S^{2} V_{1} \otimes V_{2}$ с подпространством в $V_{1} \otimes V_{1} \otimes V_{2}$ и $S^{2} V_{1}^{*} \otimes V_{2}^{*}$ с подпространством в $V_{1}^{*} \otimes V_{1}^{*} \otimes V_{2}^{*}$. Далее будем пользоваться конструкциями из [2, разд. 2]. Обозначим через $\mathfrak{g}$ алгебру Ли типа $E_{8}$, реализованную, как в [2]. Согласно конструкции из [2] мы имеем градуировку по модулю $3: \mathfrak{g}=\mathfrak{g}_{-1} \oplus \mathfrak{g}_{0} \oplus \mathfrak{g}_{1}$, где $\mathfrak{g}_{-1}=\wedge^{3} V^{*}, \mathfrak{g}_{0}=\operatorname{Lie}(\mathrm{SL}(V)), \mathfrak{g}_{1}=\wedge^{3} V$.

Рассмотрим следующее векторное пространство

$$
\mathfrak{g}^{(3)}=\mathfrak{g}_{-1}^{(3)} \oplus \mathfrak{g}_{0}^{(3)} \oplus \mathfrak{g}_{1}^{(3)},
$$

где $\mathfrak{g}_{-1}^{(3)}=S^{2} V_{1}^{*} \otimes V_{2}^{*}, \mathfrak{g}_{0}^{(3)}=\operatorname{Lie}(H), \mathfrak{g}_{1}^{(3)}=S^{2} V_{1} \otimes V_{2}$. Обозначим через $\varphi_{i}$ (соответственно $\left.\varphi_{i}^{*}\right), i=1,2$, вложение $V_{1}$ (соответственно $\left.V_{1}^{*}\right)$ в $V\left(\right.$ соответственно $V^{*}$ ) в качестве $i$-го слагаемого. Согласно [2] $\mathfrak{g}^{(3)}$ отождествляется с градуированным подпространством в $\mathfrak{g}$ следующим образом. Тензорное произведение $V_{1} \otimes V_{1} \otimes V_{2}$ может быть отождествлено с подпространством в $\wedge^{3} V$ по правилу

$$
v_{1} \otimes v_{2} \otimes v_{3}=\varphi_{1}\left(v_{1}\right) \wedge \varphi_{2}\left(v_{2}\right) \wedge v_{3}, \quad v_{1}, v_{2} \in V_{1}, \quad v_{3} \in V_{2} .
$$

Работа выполнена при поддержке Российского фонда фундаментальных исследований, грант № 98-01-00598, и CRDF, грант Rm1-2088. 
Аналогично, $V_{1}^{*} \otimes V_{1}^{*} \otimes V_{2}^{*}$ может быть отождествлено с подпространством в $\wedge^{3} V^{*}$.

Так как мы рассматриваем $U$ как подпространство в $V_{1} \otimes V_{1} \otimes V_{2}$, то и $U$ отождествляется с подпространством в $\wedge^{3} V$. Аналогично, $U^{*}=S^{2} V_{1}^{*} \otimes V_{2}^{*}$ отождествляется с подпростраснтвом в $\wedge^{3} V^{*}$. Алгебра Ли $\operatorname{Lie}(H)$ вкладывается в $\operatorname{Lie}(\operatorname{SL}(V))$ так, что элементу $(A, B) \in \operatorname{Lie}(H)$ соответствует блочно-диагональная матрища с блоками $A, A, B$.

Из правил умножения в $\mathfrak{g}\left(\right.$ см. $\left[2\right.$, п. 2.2]) следует, что $\mathfrak{g}^{(3)}$ - градуированная подалгебра алгебры $\mathfrak{g}$ с указанньпи вьше градуирующими подпространствами. Анализируя систему корней $\mathfrak{g}^{(3)}$ относительно максимального тора в $H$, получаем, что $\mathfrak{g}^{(3)}$ есть простая алгебра Ли типа $F_{4}$.

Напомним основные определения и факты из [1] применительно к представлению $H$ в $U$. Элемент пространства $U$ называется полупростым, если его $H$-орбита замкнута в $U$, и нильпотентным, если замыкание его $H$-орбиты содержит нуль. Каждый элемент $u$ из $U$ может быть однозначно представлен в виде $u=p+e$, где $p$ - полупростой, a $e$ - нильпотентный элементы из $U$ и $[p, e]=0$, где $[\cdot, \cdot]-$ умножение в $\mathfrak{g}^{(3)}$. Такое разложение называется разложением Жордана. Элемент $p$ называется полупростой, а $е$ - нильпотентной частью элемента $u$.

Классификация элементов из $U$ осуществляется методом, описанньм в [2, разд. 1]. А именно, сначала мы описьваем полупростые элементы. Они делятся на четыре семейства в соответствии с типом стабилизатора и подпространством элементов из $U$, коммутирующих с данным элементом в смысле умножения в алгебре $\mathfrak{g}^{(3)}$. Любой элемент из $U$ считается принадлежащим к семейству, содержащему его полупростую часть. После чего мы описьваем все возможные нильпотентные части для каждого семейства элементов по модулю действия стабилизатора полупростого элемента данного семейства. Это и дает классификацию элементов из $U$.

Далее, согласно $\left[2\right.$, п. 2.6] $\mathfrak{g}$ содержит однородную подалгебру типа $D_{4}$. Она описывается следующим образом. Пусть даны трехмерное комплексное векторное пространство $N$ и изоморфизмы

$$
\tau_{i}: N \rightarrow V_{i}, \quad i=1,2
$$

Тогда мы можем отождествить $S^{3} N$ с подпространством в $\wedge^{3} V$, полагая для $n_{1}, n_{2}$, $n_{3} \in N$

$$
n_{1} n_{2} n_{3}=\sum \varphi_{1}\left(\tau_{1}\left(n_{i_{1}}\right)\right) \wedge \varphi_{2}\left(\tau_{1}\left(n_{i_{2}}\right)\right) \wedge \tau_{2}\left(n_{i_{3}}\right)
$$

где сумма берется по всем перестановкам $\left(i_{1}, i_{2}, i_{3}\right)$. Аналогично пространство $S^{3} N^{*}$ может быть отождествлено с подпространством в $\wedge^{3} V^{*}$. Также мы можем рассматривать $\operatorname{Lie}(\mathrm{SL}(N))$ как диагональную подалгебру в прямой сумме $\operatorname{Lie}(H)=\operatorname{Lie}\left(\operatorname{SL}\left(V_{1}\right)\right) \oplus$ $\mathrm{Lie}\left(\mathrm{SL}\left(V_{2}\right)\right)$ и, следовательно, как подалгебру в $\mathrm{Lie}(\mathrm{SL}(V))$.

Из правил умножения в $\mathfrak{g}$ видно, что подпространство

$$
\mathfrak{g}^{(2)}=S^{3} N^{*} \oplus \operatorname{Lie}(\operatorname{SL}(N)) \oplus S^{3} N
$$

есть однородная подалгебра в $\mathfrak{g}^{(3)}$ и, следовательно, в $\mathfrak{g}$ с градуирующими подпространствами

$$
\mathfrak{g}_{-1}^{(2)}=S^{3} N^{*}, \quad \mathfrak{g}_{0}^{(2)}=\operatorname{Lie}(\operatorname{SL}(N)), \quad \mathfrak{g}_{1}^{(2)}=S^{3} N .
$$

Более того, $\mathfrak{g}^{(2)}$ есть полупростая часть централизатора (в $\left.\mathfrak{g}\right)$ полупростого элемента $\alpha P_{1}+\beta\left(P_{3}-P_{4}\right)$ (определение $P_{1}, P_{3}, P_{4}$ приведено ниже в п. 3 ), где $\alpha, \beta$ - числа такие, 
что $\alpha \beta\left(\alpha^{3}-\beta^{3}\right)\left(\alpha^{3}+8 \beta^{3}\right) \neq 0$ (см. [2, п. 5.3]). Следовательно, ограничение присоединенного представления алгебры $\mathfrak{g}^{(2)}$ на $\mathrm{SL}(N)$ и $\mathfrak{g}_{1}^{(2)}$ есть представление $\theta$-группы [1, № 26 в таблице].

Известно также (см. [2, п. 2.5]), что в $\mathfrak{g}$ содержится однородная подалгебра $\mathfrak{g}^{(1)}$ типа $E_{6}$. Она реализована следующим образом. Рассмотрим векторное пространство

$$
\begin{gathered}
\mathfrak{g}^{(1)}=\mathfrak{g}_{-1}^{(1)} \oplus \mathfrak{g}_{0}^{(1)} \oplus \mathfrak{g}_{1}^{(1)}, \\
\mathfrak{g}_{-1}^{(1)}=V_{1}^{*} \otimes V_{1}^{*} \otimes V_{2}^{*}, \quad \mathfrak{g}_{0}^{(1)}=\operatorname{Lie}\left(\mathrm{SL}\left(V_{1}\right) \times \mathrm{SL}\left(V_{1}\right) \times \mathrm{SL}\left(V_{2}\right)\right), \quad \mathfrak{g}_{1}^{(1)}=V_{1} \otimes V_{1} \otimes V_{2} .
\end{gathered}
$$

Оно отождествляется с подпространством в $\mathfrak{g}$ аналогично тому, как это сделано для $\mathfrak{g}^{(3)}$. Из [2] известно, что это подпространство есть однородная подалгебра Ли типа $E_{6}$.

Из описанных конструкций видно, что

$$
\mathfrak{g} \supset \mathfrak{g}^{(1)} \supset \mathfrak{g}^{(3)} \supset \mathfrak{g}^{(2)} .
$$

Сформулируем основные результаты. Для этого в каждом из пространств $V_{1}, V_{2}$ выберем базис. Пусть $\left\{e_{1}, e_{2}, e_{3}\right\},\left\{e_{4}, e_{5}, e_{6}\right\}$ - две копии одного и того же базиса в $V_{1}$, $\left\{e_{7}, e_{8}, e_{9}\right\}-$ базис в $V_{2}$. Положим

$$
\begin{aligned}
& u_{1}=e_{1} \otimes e_{4} \otimes e_{7}+e_{2} \otimes e_{5} \otimes e_{8}+e_{3} \otimes e_{6} \otimes e_{9}, \\
& u_{2}=e_{1} \otimes e_{5} \otimes e_{9}+e_{2} \otimes e_{6} \otimes e_{7}+e_{3} \otimes e_{4} \otimes e_{8}, \\
& u_{3}=e_{1} \otimes e_{6} \otimes e_{8}+e_{2} \otimes e_{4} \otimes e_{9}+e_{3} \otimes e_{5} \otimes e_{7} .
\end{aligned}
$$

Тогда каждый полупростой элемент эквивалентен элементу следующего вида:

$$
u=a_{1} u_{1}+a_{2}\left(u_{2}+u_{3}\right) .
$$

При этом коэффициенты $a_{1}, a_{2}$ определены с точностью до линейного преобразования из группы порядка 72 , которая порождена комплексными отражениями порядка 3 (см. [1, № 17 в таблище]).

Ниже описываются семейства элементов из $U$. В таблицах 1,2 использованы следующие обозначения. Элемент $e_{i} \otimes e_{j} \otimes e_{k}$ обозначается через $i j k$, элемент $e_{i} \otimes e_{j} \otimes e_{k}+$ $e_{p} \otimes e_{q} \otimes e_{r}-$ через $i j k p q r$ и т. д. для любого числа членов. "Представитель" - элемент, являющийся представителем класса нильпотентных частей; $\operatorname{dim} S-$ размерность стабилизатора элемента из $U$ с данной полупростой и нильпотентной частями, вычисленная по формуле из [2, п. 4.5]. Последняя строка в таблицах 1,2 соответствует нулевому элементу.

Первое семейство. Это 2-параметрическое семейство полупростых элементов. Канонический вид элемента этого семейства имеет форму (1), где коэффициенты $a_{1}, a_{2}$ таковы, что

$$
a_{1} a_{2} \neq 0, \quad a_{1}^{3}+8 a_{2}^{3} \neq 0, \quad a_{1}^{3}-a_{2}^{3} \neq 0 .
$$

Стабилизатор элемента этого семейства - абелева группа порядка 27, изоморфная $\left(\mathbb{Z}_{3}\right)^{3}$. Непосредственное вычисление показьвает, что в этом случае возможна только нулевая нильпотентная часть (см. п. 6). Элементы первого семейства составляют открытое по Зарисскому подмножество в $U$. 
ТАБЛИЦА 1

\begin{tabular}{|c|l|c|}
\hline № & Представитель & $\operatorname{dim} S$ \\
\hline 1 & 147258 & 0 \\
2 & 147 & 1 \\
3 & & 2 \\
\hline
\end{tabular}

ТАБЛИЦА 2

\begin{tabular}{|c|l|c|}
\hline № & Представитель & $\operatorname{dim} S$ \\
\hline 1 & 159249267357 & 0 \\
2 & 159249 & 1 \\
3 & & 2 \\
\hline
\end{tabular}

Второе семейство. Полупростой элемент этого семейства имеет канонический вид

$$
u=a\left(u_{2}+u_{3}\right), \quad \text { где } a \neq 0 .
$$

Коэффициент $a$ определен с точностью до умножения на корень шестой степени из единицы. Классификация возможных нильпотентных частей приведена в таблице 1.

Третье семейство. Канонический вид полупростого элемента

$$
u=a u_{1}, \quad \text { где } a \neq 0 .
$$

Коэффициент $a$ определен с точностью до умножения на корень шестой степени из единицы. Классификация возможных нильпотентных частей приведена в таблище 2.

Четвертое семейство. Здесь $u=0$. Классификация возможных нильпотентных частей есть просто классификация нильпотентных элементов в $U$. Она приведена в таблице 3 (см. п. 4).

Описание образующих алгебры инвариантов приведено в п. 5.

3. Согласно [1] рассматриваемое представление совпадает с представлением $\theta$-группы (см. [1, № 17 в таблице]), дифференциал которого есть присоединенное представление $\mathfrak{g}_{0}^{(3)}$ в $\mathfrak{g}_{1}^{(3)}$. Из свойств $\theta$-групा следует, что в пространстве данного представления имеется картановское подпространство. Для его описания рассмотрим элементы

$$
\begin{gathered}
P_{i}=e_{k_{i}^{1}} \wedge e_{j_{i}^{1}} \wedge e_{l_{i}^{1}}+e_{k_{i}^{2}} \wedge e_{j_{i}^{2}} \wedge e_{l_{i}^{2}}+e_{k_{i}^{3}} \wedge e_{j_{i}^{3}} \wedge e_{l_{i}^{3}}, \\
P_{i}^{*}=e^{k_{i}^{1}} \wedge e^{j_{i}^{1}} \wedge e^{l_{i}^{1}}+e^{k_{i}^{2}} \wedge e^{j_{i}^{2}} \wedge e^{l_{i}^{2}}+e^{k_{i}^{3}} \wedge e^{j_{i}^{3}} \wedge e^{l_{i}^{3}}
\end{gathered}
$$

где $i$ - номер одного из следующих наборов:

1) $(123,456,789)$;

2) $(147,258,369)$;

3) $(159,267,348)$;

4) $(168,249,357)$;

$k_{i}^{n}, j_{i}^{n}, l_{i}^{n}-n$-я тройка чисел из $i$-го набора, а верхние индексы у $e$ нумеруют двойственньй базис сопряженного пространства. Из определения пространств $\mathfrak{g}_{-1}^{(3)}, \mathfrak{g}_{1}^{(3)}$ и их вложений в $\mathfrak{g}_{-1}, \mathfrak{g}_{1}$ соответственно следует, что $P_{2}, P_{3}+P_{4} \in \mathfrak{g}_{1}^{(3)}$, причем $P_{2}=u_{1}$, $P_{3}=u_{2}, P_{4}=u_{3}$, а $P_{2}^{*}, P_{3}^{*}+P_{4}^{*} \in \mathfrak{g}_{-1}^{(3)}$. Вследствие леммы из [2, разд. 3], если $x \in \mathfrak{g}_{1}^{(3)}$ коммутирует с $P_{2}$, то он коммутирует с $P_{2}^{*}$. Используя те же рассуждения, что и в доказательстве этой леммы, можно показать, что если $x \in \mathfrak{g}_{1}^{(3)}$ коммутирует с $P_{3}+P_{4}$, то он коммутирует с $P_{3}^{*}+P_{4}^{*}$. Так как $P_{2}, P_{3}+P_{4}, P_{2}^{*}, P_{3}^{*}+P_{4}^{*}$ друг с другом коммутируют и полупросты (см. [2]), то подпространство $\mathscr{L}:=\left\langle P_{2}, P_{3}+P_{4}, P_{2}^{*}, P_{3}^{*}+P_{4}^{*}\right\rangle$ есть подалгебра Картана алгебры $\mathfrak{g}^{(3)}$ и $\mathscr{L}^{(3)}:=\left\langle P_{2}, P_{3}+P_{4}\right\rangle-$ картановское подпространство 
в $\mathfrak{g}_{1}^{(3)}$. Следовательно, любой полупростой элемент из $U$ эквивалентен линейной комбинации элементов $u_{1}, u_{2}+u_{3}$. Из свойств $\theta$-групп (см. [1]) следует, что мы получили описание полупростых элементов с точностью до действия конечной групы (групшы Вейля градуированной алгебры $\left.\mathfrak{g}^{(3)}\right)$, указанной в [1, № 17 в таблице]. Обозначим эту группу через $W^{(3)}$. Опишем $W^{(3)}$ геометрически.

Заметим, что элементы

$$
(\omega E, E),\left(\omega^{2} E, E\right) \in H, \quad \text { где } \omega^{3}=1
$$

переводят $\mathscr{L}^{(3)}$ в себя и индуцируют в $\mathscr{L}^{(3)}$ операторы умножения на $\omega^{2}, \omega$ соответственно.

Как известно из $[1$, п. 9.3$]$, группа Вейля однородной подалгебры $\mathfrak{g}^{(2)}$, являющейся полупростой частью централизитора элемента $\alpha P_{1}+\beta\left(P_{3}-P_{4}\right)$ в $\mathfrak{g}($ см. [2, таблища 7]), есть стабилизатор $W_{\alpha P_{1}+\beta\left(P_{3}-P_{4}\right)}$ элемента $\alpha P_{1}+\beta\left(P_{3}-P_{4}\right)$ в групе Вейля градуированной алгебры $\mathfrak{g}$. С другой стороны, $\mathfrak{g}^{(2)}$ является однородной подалгеброй в $\mathfrak{g}^{(3)}$, и их ранги как ранги градуированных алгебр равны [1]. Поэтому (см. [1, п. 9.3]) группа Вейля градуированной алгебры $\mathfrak{g}^{(2)}$ изоморфнна подгрупше групшы Вейля градуированной алгебры $\mathfrak{g}^{(3)}$. Прямые вычисления показывают, что группа $W^{(3)}$ порождается подгруппой $W_{\alpha P_{1}+\beta\left(P_{3}-P_{4}\right)}$ и умножениями на $\omega, \omega^{2}$ (см. [3, таблица 1$\left.]\right)$. Следовательно, из сказанного в $[2$, п. 3.3$]$ и в $[3$, п. 1.4$]$ получаем, что зеркала отражений из групшы Вейля $W^{(3)}$ - это прямые, порожденные векторами $u_{1}, u_{1}+u_{2}+u_{3}, u_{1}+\omega\left(u_{2}+u_{3}\right)$, $u_{1}+\omega^{2}\left(u_{2}+u_{3}\right), u_{2}+u_{3},-2 u_{1}+u_{2}+u_{3},-2 \omega^{2} u_{1}+u_{2}+u_{3},-2 \omega u_{1}+u_{2}+u_{3}$, и этими отражениями порождается указанная группа. Заметим, что приведенные вьше векторы - это в точности собственные векторы отражений из групшы $W_{\alpha P_{1}+\beta\left(P_{3}-P_{4}\right)}(\mathrm{cm}$. [3, п. 1.4]).

4. Классификация нильпотентных элементов изучаемого представления получена при помощи метода характеристик, изложенного в [2, разд. 4]. Результат вычислений приводится в таблице 3 .

В таблице 3 использованы следующие обозначения. Так $\operatorname{dim} S$ - размерность стабилизатора, вычисленная по формуле, указанной в [2, п. 4.5]. Элемент $e_{i} \otimes e_{j} \otimes e_{k}$ обозначен через $i j k$, элемент $e_{i} \otimes e_{j} \otimes e_{k}+e_{p} \otimes e_{q} \otimes e_{r}-$ через $i j k p q r$ и т.д. для любого числа членов. "Представитель" - элемент, являющийся представителем класса нильпотентньх элементов. “Тип” - тип неприводимого нильпотентного элемента в смысле [2, п. 4.2]. Последняя строка соответствует нулевому элементу. “Тип $S$ " - тип редуктивной части стабилизатора “представителя". И наконец, $\mathbb{T}^{n}-n$-мерньй алгебраический тор.

5. Как известно (см. [1, № 17 в таблице]), алгебра инвариантов рассматриваемого представления порождается двумя свободными однородными образующими степеней 6,12 . Следовательно, полную систему свободньх образующих будут составлять любые два однородных инварианта $F_{6}, F_{12}$ этих степеней, не удовлетворяющие соотношению типа

$$
\lambda F_{6}^{2}=F_{12}
$$

где $\lambda$ - комплексное число.

Обозначим форму объема пространства $V_{i}$ через $\varepsilon(i)_{i_{1} i_{2} i_{3}}$. Будем искать инвариант степени $3 k$ в виде полной свертки тензорного произведения $3 k$ элементов из $U, 2 k$ форм объема пространства $V_{1}$ и $k$ форм объема пространства $V_{2}$. Индексам формы объема 
ТАБЛИЦА 3

\begin{tabular}{|c|l|c|l|l|}
\hline № & \multicolumn{1}{|c|}{ Представитель } & $\operatorname{dim} S$ & \multicolumn{1}{|c|}{ Тип } & Тип $S$ \\
\hline 1 & 147259268358169349 & 0 & $F_{4}$ & 0 \\
2 & 147268358159249257 & 1 & $C_{3}+A_{1}$ & 0 \\
3 & 147259367268358 & 1 & $B_{4}$ & 0 \\
4 & 147268358159249 & 2 & $C_{3}$ & $\mathbb{T}^{1}$ \\
5 & 147258169349 & 2 & $B_{3}$ & $\mathbb{T}^{1}$ \\
6 & 147257258368 & 2 & $B_{2}+2 A_{1}$ & 0 \\
& & & $D_{4}\left(a_{1}\right)$ & \\
& & & $2 A_{2}$ & \\
7 & 147258159249267357 & 3 & $2 A_{2}$ & 0 \\
8 & 147268358257 & 3 & $B_{2}+A_{1}$ & $\mathbb{T}^{1}$ \\
9 & 157247168348367 & 4 & $A_{2}+A_{1}$ & $\mathbb{T}^{1}$ \\
10 & 147258367 & 4 & $B_{2}$ & $\mathbb{T}^{2}$ \\
11 & 157247168348149 & 5 & $A_{2}+A_{1}$ & $A_{1}$ \\
12 & 147258168348 & 5 & $A_{2}+A_{1}$ & $\mathbb{T}^{1}$ \\
13 & 147258159249 & 5 & $A_{2}+A_{1}$ & $A_{1}$ \\
14 & 157247168348 & 6 & $A_{2}$ & $A_{1}+\mathbb{T}^{1}$ \\
15 & 147258 & 6 & $A_{2}$ & $\mathbb{T}^{2}$ \\
16 & 147158248 & 7 & $2 A_{1}$ & $\mathbb{T}^{2}$ \\
17 & 147257367 & 8 & $2 A_{1}$ & $2 A_{1}$ \\
18 & 147257 & 11 & $A_{1}$ & $A_{1}+\mathbb{T}^{2}$ \\
19 & 147 & & & $2 A_{1}+\mathbb{T}^{1}$ \\
20 & & & & \\
\hline
\end{tabular}

$i$-го пространства при свертке могут соответствовать только индексы, стоящие на первом или втором месте у элементов из $U$, если $i=1$, или стоящие на третьем месте у элементов из $U$, если $i=2$.

Согласно $[1$, № 2 в таблище] естественное представление групшы

$$
G_{0}^{(1)}=\mathrm{SL}\left(V_{1}\right) \times \mathrm{SL}\left(V_{1}\right) \times \mathrm{SL}\left(V_{2}\right)
$$

в пространстве $U^{(1)}=V_{1} \otimes V_{1} \otimes V_{2}$ есть $\theta$-группа и ограничение его на $H$ и $U$ есть изучаемое представление. Заметим, что $H$ вкладывается в $G_{0}^{(1)}$ аналогично своей алгебре Ли. Пусть $a_{1}, a_{2}, a_{3}$ - координатные функции относительно базиса $u_{1}, u_{2}, u_{3}$ на картановском подпространстве $\mathscr{L}^{(1)}=\left\langle u_{1}, u_{2}, u_{3}\right\rangle$ для представления $G_{0}^{(1)}$ в $U^{(1)}$ (см. [4, п. 3] $]^{1}$ ). Тогда согласно описанию $\mathscr{L}^{(3)}$ (см. п. 3) картановское подпространство $\mathscr{L}^{(3)}$ для представления $H$ в $U$ есть подпространство в $\mathscr{L}^{(1)}$, заданное уравнением $a_{2}=a_{3}$.

\footnotetext{
${ }^{1} \mathrm{~B}$ работе [4, п. 2, таблица 2] допущена неточность. Описание возможных нильпотентных частей третьего семейства получается добавлением к элементам, указанным в таблице 2, элементов, получаемых из "представителей" под номерами 1-4 в таблице 2 при помощи процедуры, указанной в $[4$, п. 4$]$.
} 
Понятно, что ограничения инвариантов действия $G_{0}^{(1)}$ на $U^{(1)}$ на $U$ будут давать инварианты изучаемого представления. Из общей теории $\theta$-групп известно, что алгебра инвариантов действия $G_{0}^{(1)}$ на $U^{(1)}$ (соответственно $H$ на $U$ ) изоморфна путем ограничения инвариантов алгебре инвариантов действия $W^{(1)}$ на $\mathscr{L}^{(1)}\left(\right.$ соответственно $W^{(3)}$ на $\left.\mathscr{L}^{(3)}\right)$; здесь $W^{(1)}$ - группа Вейля градуированной алгебры $\mathfrak{g}^{(1)}$. Заметим, что гомоморфизм ограничения с $U^{(1)}$ на $U$, упомянутый вьше, индуцирует гомоморфизм алгебры инвариантов действия $W^{(1)}$ на $\mathscr{L}^{(1)}$ в алгебру инвариантов действия $W^{(3)}$ на $\mathscr{L}^{(3)}$.

Согласно [5, гл. 4, п. 7] алгебра инвариантов действия $W^{(1)}$ на $\mathscr{L}^{(1)}$ содержит следующие образуюшие степеней 6,12 :

$$
\begin{gathered}
F_{6}=\left(a_{1}^{3}+a_{2}^{3}+a_{3}^{3}\right)^{2}-12\left(a_{1}^{3} a_{2}^{3}+a_{1}^{3} a_{3}^{3}+a_{2}^{3} a_{3}^{3}\right), \\
F_{12}=\left(a_{1}^{3}+a_{2}^{3}+a_{3}^{3}\right)^{4}+216\left(a_{1}^{3}+a_{2}^{3}+a_{3}^{3}\right) a_{1}^{3} a_{2}^{3} a_{3}^{3} .
\end{gathered}
$$

Непосредственная проверка показывает, что ограничения этих образуюших на $\mathscr{L}^{(3)}$ не удовлетворяют соотношению типа (2).

Следовательно, ограничение образующих алгебры инвариантов степеней 6,12 действия $G_{0}^{(1)}$ на $U^{(1)}$ на $U$ дает инварианты, не удовлетворяюшие соотношению типа (2). В результате получаем, что алгебра инвариантов действия $H$ на $U$ есть образ (при ограничении с $U^{(1)}$ на $U$ ) алгебры инвариантов действия $G_{0}^{(1)}$ на $U^{(1)}$. Образуюшие алгебры инвариантов изучаемого представления суть ограничения инвариантов степеней 6,12 , указанных в $[4$, п. 5$]$.

6. Разделение полупростых элементов на семейства производится так же, как в [2, п. 3.4], т.е. все сводится к классам сопряженности стабилизаторов элементов картановского подпространства в групе Вейля $W^{(3)}$, а их описания получаются несложными вычислениями с отражениями. Вместе с тривиальными классами их четыре. Отметим, что имеется восемь зеркал отражений и две орбиты группы $W^{(3)}$ в множестве всех этих зеркал. Компонента связности стабилизаторов полупростых элементов второго и третьего семейств, содержащая единичньй элемент, - это двумерньй тор (всего у стабилизаторов три компоненты связности). Используя аргументы из [2, п. 3.4 и разд. 5], можно легко получить указанную в п. 2 классификацию семейств полупростых элементов и нильпотентных частей для каждого из них.

Авторы благодарны своему научному руководителю Э.Б. Винбергу за постановку задачи и постоянное внимание к работе.

\section{СПИСОК ЦИТИРОВАННОЙ ЛИТЕРАТУРЫ}

[1] Винберг Э. Б. Группа Вейля градуированной алгебры Ли // Изв. АН СССР. Сер. матем. 1976. Т. 40. №3. С. 488-526.

[2] Винберг Э.Б., Элашвили А. Г. Классификация тривекторов девятимерного пространства // Тр. семинара по векторному и тензорному анализу. Т. 18. М.: Изд-во МГУ, 1978. C. 197-233.

[3] Shephard G.C., Todd J.A. Finite unitary reflection groups // Canad. J. Math. 1954. V. 6. № 2. P. 274-304.

[4] Нурмиев А. Г. Орбиты и инварианты кубических матриц третьего порядка // Матем. сб. 2000. T. 191. № 5. C. 101-108.

[5] Спрингер Т. Теория инвариантов. М.: Мир, 1981. 